\title{
Synthesis and Characterization of Platinum(II) Complexes from Trifluoromethyl Phenylenediamine, Picoline and $N$-Benzyl Ethylenediamine Derivatives
}

\author{
Mauro V. de Almeida, ${ }^{*, a}$ Joana Darc S. Chaves, ${ }^{a}$ Ana Paula Soares Fontes, ${ }^{a}$ \\ Eloi T. César ${ }^{b}$ and Marcel Gielen ${ }^{c}$ \\ ${ }^{a}$ Departamento de Química, Universidade Federal de Juiz de Fora, 36036-900 Juiz de Fora-MG, Brazil \\ ${ }^{b}$ Colégio de Aplicação João XXIII, Juiz de Fora-MG, Brazil \\ ${ }^{c}$ Faculty of Applied Sciences, Free University of Brussels, B-1050 Brussels, Belgium
}

\begin{abstract}
Este trabalho descreve a síntese e a caracterização de onze novos complexos de platina(II), contendo como ligantes derivados da 1,2-fenilenodiamina, da $\mathrm{N}$-benzil etilenodiamina, e da 2ou 4-picolina. Os complexos foram preparados em rendimentos satisfatórios pela reação dos ligantes com $\mathrm{K}_{2}\left[\mathrm{PtCl}_{4}\right]$. É descrita também a atividade citotóxica de um dos complexos de platina(II), em sete linhagens de células tumorais de origem humana, que se mostrou muito menos ativo do que a cisplatina.
\end{abstract}

This work describes the synthesis and characterization of eleven new platinum(II) complexes having ligands derived from 1,2-phenylenediamine, $\mathrm{N}$-benzyl ethylenediamine and 2- or 4picoline. The complexes were prepared in satisfactory yields by reaction of $\mathrm{K}_{2}\left[\mathrm{PtCl}_{4}\right]$ with the appropriate ligand. The cytotoxic pre-screening of one of these complexes in seven human cancer cell lines showed that this compound is much less active than cisplatin.

Keywords: platinum(II) complexes, 1,2-phenylenediamine, $N$-benzyl ethylenediamine, picoline, cancer

\section{Introduction}

The discovery of the anticancer activity of the coordination compound cis-diaminedichloroplatinum(II)

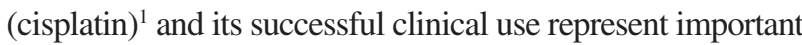
advances for inorganic medicinal chemistry. Cisplatin is one of the most widely used and effective oncological agents against cancers of the testicles, ovaries, bladder, head and neck. ${ }^{24}$ However, its clinical usefulness has frequently been limited by severe side effects, such as nephrotoxicity, ototoxicity and neurotoxicity and the emergence of drug resistance..$^{5-7}$ Thus, thousands of platinum complexes have been synthesized, aiming to find drugs that have a broader spectrum of activity than that of cisplatin, and reduced side effects. The majority of them followed the set of structureactivity relationships summarized by Cleare and Hoeschele, ${ }^{8,9}$ which states that the main condition for a platinum complex to show antitumour activity is that it should have a cis geometry with the general formula cis-[ $\left.\operatorname{PtX}_{2}(\mathrm{Am})_{2}\right]$, where

* e-mail: mvieira@quimica.ufjf.br
$\mathrm{X}$ is the leaving group ( $\mathrm{Cl}$ is the most frequently used) and $\mathrm{Am}$ is an inert amine (mono or bidentate) with at least one $\mathrm{N}-\mathrm{H}$ moiety.

According to Bednarski, ${ }^{10}$ the "chelate effect" is another important point that must be considered in the synthesis of platinum complexes that contain diamine and related compounds as non-leaving linkers. In the organism, cisplatin can react with some biological molecules such as glutathione, leading to the replacement of the amine group, which could result in inactivity of the complex. For platinum(II) complexes with diamine ligands, this reaction would be less favored due to their higher stability as compared to monodentate nitrogen ligands. For example, dichloro(o-phenylenediamine)platinum(II) complex (Figure 1 , a) was among the first cisplatin analogs found to possess antitumour activity. ${ }^{11-14}$

Thus, Connors et al. ${ }^{11}$ have prepared different platinum(II) complexes which contains aromatic amines, such as 1,2phenylenediamine and its derivatives, having different groups in the 4 position, such as chloro, methoxy, nitro and methyl. Later, Meischen et al. ${ }^{15}$ reported the antineoplastic activity 
of a large number of complexes, such as: cis-dichloro (4chloro-1,2-phenylenediamine)platinum(II) (Figure 1, b) and cis-dichloro (1,2-phenylenediamine)platinum(II) (Figure 1, a). Although these compounds were less active than cisplatin, they have shown relevant biological activity against the L1210 leukemic cell line.

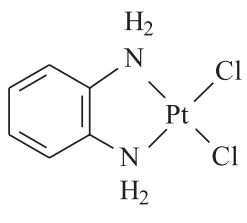

a

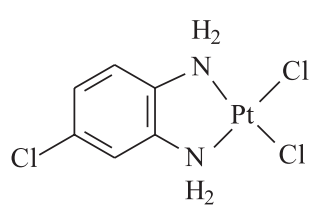

b
Figure 1. Platinum(II) complexes which present biological activity: cisdichloro (4-chloro-1,2-phenylenediamine)platinum(II) a and dichloro (1,2-phenylenediamine)platinum(II) b.

It is also important to emphasize that several compounds containing the pyridine ring in their structure are quite important in the medical and biological fields. As examples, one can mention vitamin B6 (pyridoxine), nicotine and chlorpheniramine. Some pyridines can act as painkillers, anti-hystaminic and heart stimulants. They also can show antineoplastic activity in vitro in the cells of skin carcinoma. ${ }^{16}$

Meischen et al. ${ }^{15}$ have described the synthesis of many platinum(II) complexes which contain pyridine and picoline derivatives as ligands that showed antineoplasic activity in leukemia lymphoma.

Considering that complexes having diamines as chelated ligands have shown activity against several types of tumors ${ }^{17,18}$ and that many aromatic compounds have demonstrated the ability to intercalate between DNA bases, ${ }^{19}$ we have recently reported the synthesis of a series of platinum(II) complexes with ethylenediamine derivatives ${ }^{20}$ and 1,3-propanediamine derivatives. ${ }^{21}$ In this paper we report the preparation and characterization of cisplatin analogs having 1,2-diamines or 1,2-aminethiol as ligands, derivatives of 1,2-phenylenediamine, $N$-benzyl ethylenediamine and picoline.

\section{Results and Discussion}

Proligand $\mathbf{1}$ (Scheme 1) is commercially available and was purified before use by column chromatography (eluent: hexane/ethyl acetate).

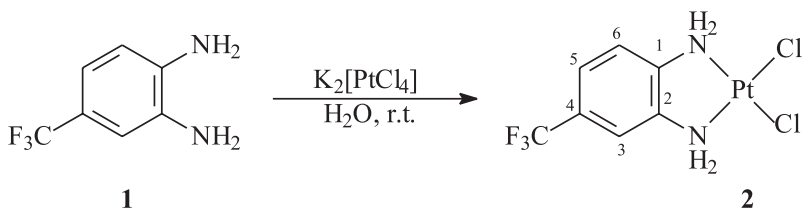

Scheme 1.
Proligands 6, 7 and $\mathbf{8}$ (Scheme 2) were prepared by treating ethylenediamine with the corresponding benzyl halide in ethanol at room temperature. In the synthesis of these proligands, we have observed the formation of $N, N^{\prime}$ dibenzylated compounds as well. Thus, during the purification process of proligand 7, we have also isolated the corresponding $N, N^{\prime}$-di-(2-nitrobenzyl)ethylenediamine $\mathbf{1 6}$.

Compounds 12 and 13 (Scheme 2) were obtained, respectively, by reduction $\left(\mathrm{H}_{2} / \mathrm{Pd}\right)$ of the proligands 6 and 7 in methanol.

Compounds 19, 20, 24 and 25 (Scheme 3) were prepared by the reaction of 2-(bromomethyl)pyridine hydrobromide and 4-(bromomethyl)pyridine hydrobromide with ethylenediamine (proligands 19 and 24) or with 2aminoethanethiol hydrochloride (proligands 20 and 25), in the presence of sodium hydrogencarbonate in ethanol.

All the prepared proligands are new compounds and were purified by column chromatography (eluent: dichoromethane/methanol) with satisfactory yields. They were characterized by analytical and spectroscopic methods (see experimental). The IR spectra of all the proligands showed the characteristic absorptions. For instance, compound 6 shows $v_{\mathrm{N}-\mathrm{H}}$ at $3218 \mathrm{~cm}^{-1}, v_{\mathrm{C}-\mathrm{H} \text { (aromatic) }}$ at $3077 \mathrm{~cm}^{-1}$ and $v_{\mathrm{C}-\mathrm{H} \text { (aliphatic) }}$ at $2936 \mathrm{~cm}^{-1}$. In addition to these absorptions, the IR spectrum of proligand 1 showed $v_{\mathrm{C}-\mathrm{F}}$ at $1332 \mathrm{~cm}^{-1}$, the IR spectrum of compounds 6, 7 and 16 showed $v_{\mathrm{N}-\mathrm{O}}$ at 1517 and $1346 \mathrm{~cm}^{-1}$; for compound $\mathbf{8}$ one can observe $v_{\mathrm{C}=\mathrm{O}}$ at $1728 \mathrm{~cm}^{-1}$. Compounds 19, 20, 24 and 25 showed $v_{\mathrm{C}=\mathrm{N}}$ at $1625 \mathrm{~cm}^{-1}$ and $v_{\mathrm{C}-\mathrm{S}}$ at $632 \mathrm{~cm}^{-1}$ (for compounds 20 and $\mathbf{2 5}$ ).

In the ${ }^{1} \mathrm{H}$ NMR spectra, signals were observed in the $\delta$ $2.8-4.0$ and $7.0-7.8$, region corresponding to the methylene groups and aromatic hydrogens, respectively. For compound $\mathbf{8}$, a signal at $\delta 3.8$ attributable to the $\mathrm{OCH}_{3}$ was also observed.

In the ${ }^{13} \mathrm{C}$ NMR spectra, signals at the $\delta 35$ - 50 region corresponding to the methylenic carbons and signals in the $\delta 115$ to 160 region, due to the aromatic carbons, were observed. For compound $\mathbf{8}$, the signals corresponding to $\mathrm{O}^{\mathrm{CH}}{ }_{3}$ and $\underline{\mathrm{C}}=\mathrm{O}$ were observed at $\delta 52.1$ and 166.2, respectively.

The dichloroplatinum(II) complexes (Schemes 2 and 3) were synthesized by adding the corresponding proligands, previously dissolved in water or methanol to a solution of $\mathrm{K}_{2}\left[\mathrm{PtCl}_{4}\right]$ in water at room temperature, and were isolated by simple filtration.

In addition to the absorptions observed for the ligands, the IR spectra of the complexes exhibited absorptions corresponding to $\mathrm{v}_{\mathrm{Pt}-\mathrm{N}}$ and $\mathrm{v}_{\mathrm{Pt}-\mathrm{Cl}}$ in the 450 and $320 \mathrm{~cm}^{-1}$ region, respectively. For all complexes, the ${ }^{1} \mathrm{H}$ NMR spectra showed resonances between $\delta$ 6.5-9.3 corresponding to the aromatic hydrogens, and large 
singlets between $\delta$ 5.2-8.5 corresponding to the $\mathrm{NH}_{2}$ group. Compound 11 also showed a signal at $\delta 3.7$ attribuited to the $\mathrm{OCH}_{3}$ group. In the ${ }^{13} \mathrm{C} \mathrm{NMR}$, signals between $\delta 113.9$ 166.6 corresponding to the aromatic carbons were observed. For complex 11, one signal at $\delta 52.2$ showed the presence of the methoxy group. The ${ }^{195} \mathrm{Pt}$ NMR spectra for complex 2 showed only one signal at $\delta-2194$. This chemical shift value can be expected based on data for similar compounds described in the literature. For instance, the spectrum of $\left[\mathrm{Pt}(\mathrm{DACH}) \mathrm{Cl}_{2}\right]$ shows a signal at $\delta-2287$ (DACH=1,2-diaminocyclohexane). ${ }^{22}$ The results of elemental analyses for the complexes are in agreement with the calculated values.

Compound $\mathbf{2}$ was selected for cytotoxic activity test to evaluate the rule of the electron-withdrawing $\mathrm{CF}_{3}$ group in the aromatic ring since analogous compounds have shown cytotoxic activity close to cisplatin. ${ }^{11-14}$ This compound was screened in vitro against seven human cancer cell lines, MCF7 and EVSAT (mammary cancers), WiDr (colon cancer), IGROV (ovarian cancer), M19 (melanoma), MEL A498 (renal cancer) and H226 (lung cancer). The $\mathrm{ID}_{50}$ values (in $\mathrm{ng} \mathrm{mL}^{-1}$ ) obtained are presented in Table 1 together with those of clinically used drugs, doxorubicin (DOX), cisplatin (CPT), 5-fluorouracil (5-FU), methotrexate (MTX), etoposide (ETO) and taxol (TAX) for comparison.

The $\mathrm{ID}_{50}$ values clearly show that compound $\mathbf{2}$ is less active in vitro than cisplatin, and much less active than doxorubicin, for instance (Table 1). This result suggests that the presence of the strong electron-withdrawing group in the aromatic ring lead to a decrease in the cytotoxic activity.

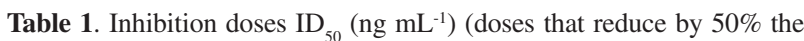
growth of the cell lines) for compound $\mathbf{2}$ and other antitumoral drugs

\begin{tabular}{lccccccc}
\hline & DOX & CPT & 5-FU & MTX & ETO & TAX & $\mathbf{2}$ \\
\hline A498 & 90 & 2253 & 143 & 37 & 1314 & $<3$ & 38445 \\
EVSAT & 8 & 422 & 475 & 5 & 317 & $<3$ & 3917 \\
H226 & 199 & 3269 & 340 & 2287 & 3934 & $<3$ & 10059 \\
IGROV & 60 & 169 & 297 & 7 & 580 & $<3$ & 15553 \\
M19 & 16 & 558 & 442 & 23 & 505 & $<3$ & 11532 \\
MCF7 & 10 & 699 & 750 & 18 & 2594 & $<3$ & 17778 \\
WiDr & 11 & 967 & 225 & $<3$ & 150 & $<3$ & 16108 \\
\hline
\end{tabular}

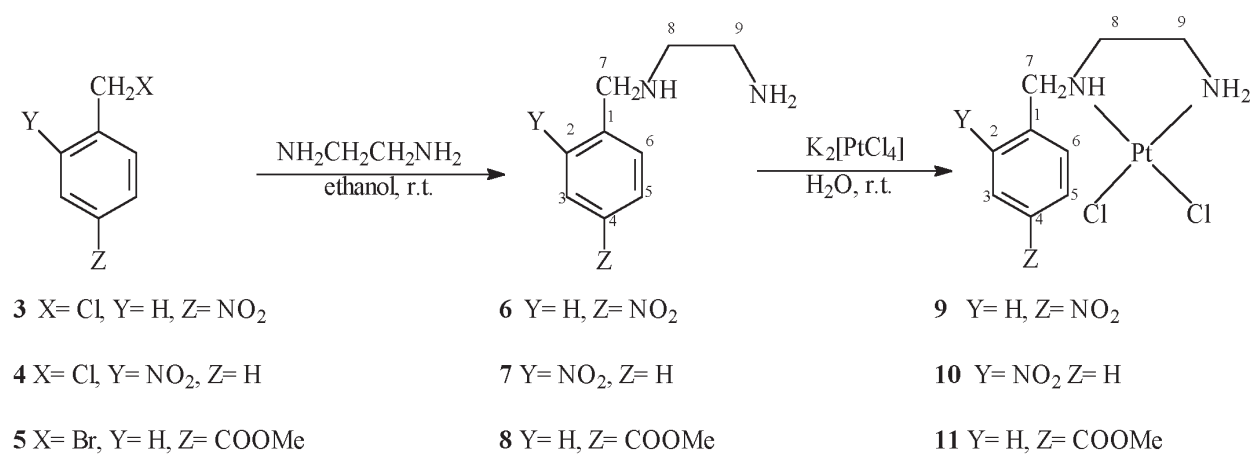

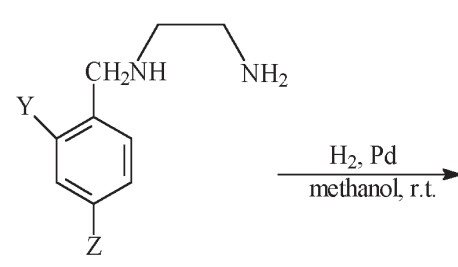

$6 \mathrm{Y}=\mathrm{H}, \mathrm{Z}=\mathrm{NO}_{2}$

$7 \mathrm{Y}=\mathrm{NO}_{2}, \mathrm{Z}=\mathrm{H}$<smiles>CCNCCNCc1ccccc1[N+](=O)[O-]</smiles>

16

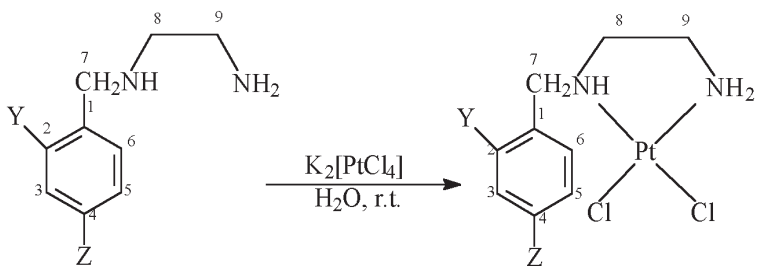

$12 \mathrm{Y}=\mathrm{H}, \mathrm{Z}=\mathrm{NH}_{2}$

$14 \mathrm{Y}=\mathrm{H}, \mathrm{Z}=\mathrm{NH}_{2}$

$13 \mathrm{Y}=\mathrm{NH}_{2}, \mathrm{Z}=\mathrm{H}$

$15 \mathrm{Y}=\mathrm{NH}_{2}, \mathrm{Z}=\mathrm{H}$

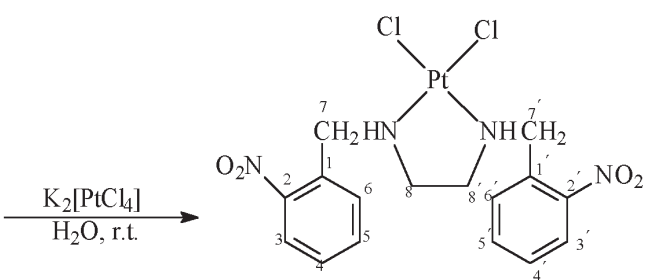

17

Scheme 2. 


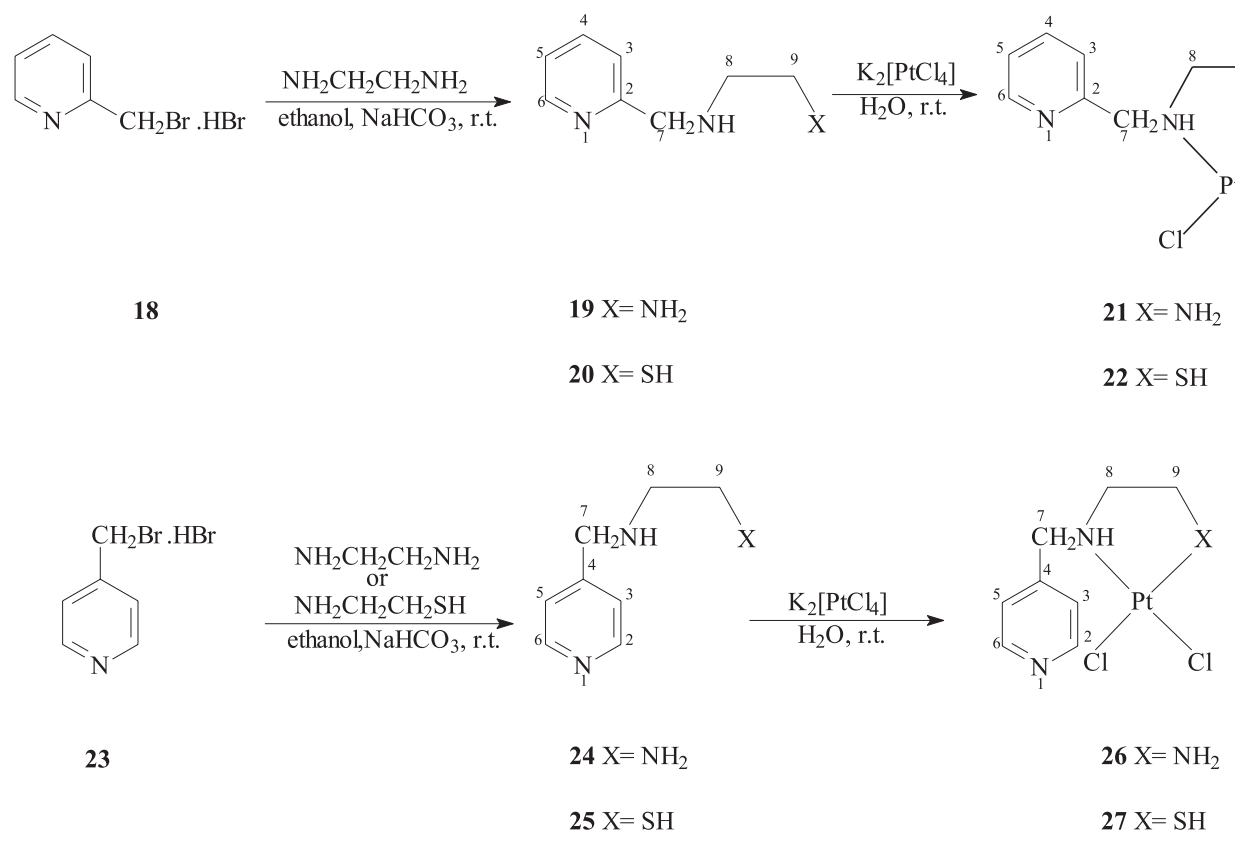

Scheme 3.

\section{Experimental}

IR spectra were obtained on a Bomem FT IR MB-102 spectrometer from $\mathrm{KBr}$ pellets. ${ }^{1} \mathrm{H}$ NMR (200 and $400 \mathrm{MHz}$ ), ${ }^{13} \mathrm{C}$ NMR (50 and $100 \mathrm{MHz}$ ) and ${ }^{195} \mathrm{Pt}$ NMR (86 MHz) spectra were recorded on a Bruker Avance DRX 200 and DRX 400 spectrometers at the Federal University of Minas Gerais. Elemental analyses were done at the Mycroanalyses Laboratory at ICNS/CNRS, Gif-sur-Yvette, France and at Central Analítica, USP-Brazil. The progress of all reactions was monitored by thin-layer chromatography which was performed on $2.0 \times 6.0 \mathrm{~cm}$ aluminium sheets precoated with silica gel 60 (HF-254, Merck) to a thickness of $0.25 \mathrm{~mm}$. The developed chromatograms were viewed under an ultraviolet light. For column chromatography Merck silica gel (70-230 mesh) was used. Solvents were purchased from Vetec Química and were distilled before use. Reagents were purchased from Aldrich and used without further purification.

Synthesis of proligands $N$-(4-nitrobenzyl)-ethylenediamine (6), $N$-(2-nitrobenzyl)-ethylenediamine) (7), N-[4-methylcarboxylate)-benzyl]-ethylenediamine (8) and $N, N^{\prime}-d i-(2-$ nitrobenzyl)ethylenediamine (16)

To ethylenediamine $(6.7 \mathrm{~mL}, 100 \mathrm{mmol})$ in ethanol (30 $\mathrm{mL}$ ), the corresponding benzyl halide ( $20 \mathrm{mmol}$ ) was slowly added during $4 \mathrm{~h}$. The reaction mixture was stirred for $24 \mathrm{~h}$ at room temperature. After this time, the solvent was evaporated under reduced pressure and the residue, purified on silica, using 9:1 dichloromethane/methanol as eluent.
Yields: 6 (2.45 g; 63\%); 7 (2.49 g; 64\%); 8 (1.64 g; 40\%). The compound $\mathbf{1 6}$ was also isolated in $14 \%$ yield during the preparation of compound 7 .

Data for 6. Oil; Elemental analysis (Found: C, 55.58; H, 6.43; N, 21.70. Calc. for $\mathrm{C}_{9} \mathrm{H}_{13} \mathrm{~N}_{3} \mathrm{O}$ : C, $55.38 ; \mathrm{H}, 6.67 ; \mathrm{N}$, 21.54\%); IR (KBr) $v_{\max } / \mathrm{cm}^{-1}: 3444,3344,3218,3077,2936$, $2844,1517,1346 ;{ }^{1} \mathrm{H}$ NMR (200 MHz, DMSO- $\left.d_{6}\right): \delta 2.75$ (t, $\left.2 \mathrm{H}, \mathrm{H} 9, J_{9-8} 5.6 \mathrm{~Hz}\right), 2.89$ (t, 2H, H8), $3.85\left(\mathrm{~s}, 2 \mathrm{H}, \mathrm{CH}_{2} \mathrm{Ph}\right.$ ), 5.02 (s, 3H, $\underline{\mathrm{H}}$ and $\left.\mathrm{N}_{2}\right), 7.68\left(\mathrm{~d}, 2 \mathrm{H}, \mathrm{H} 2, \mathrm{H} 6, J_{2-3} 8.6\right.$ $\mathrm{Hz}$ ), 8.18 (d, 2H, H3, H5); ${ }^{13} \mathrm{C}$ NMR (50MHz, DMSO- $d_{6}$ ): $\delta 40.7\left(\underline{\mathrm{CH}}_{2} \mathrm{NH}_{2}\right), 45.7\left(\underline{\mathrm{CH}}_{2} \mathrm{NH}\right), 51.3\left(\underline{\mathrm{CH}}_{2} \mathrm{Ph}\right), 123.2(\mathrm{C} 3 /$ C5), 129.0 (C2/C6), 146.3 (C1), 149.1 (C4).

Data for 7. Oil; Elemental analysis (Found: C, 55.47; H, 6.45; N, 21.80. Calc. for $\mathrm{C}_{9} \mathrm{H}_{13} \mathrm{~N}_{3} \mathrm{O}_{2}: \mathrm{C}, 55.38 ; \mathrm{H}, 6.67 ; \mathrm{N}$, 21.54\%); IR (KBr) $v_{\text {max }} / \mathrm{cm}^{-1}: 3282,3040,2968,2853,1523$, 1351; ${ }^{1} \mathrm{H}$ NMR (200 MHz, CD OD): $\delta 2.89$ (t, 2H, H9, $\left.J_{9-8} 5.8 \mathrm{~Hz}\right), 3.05(\mathrm{t}, 2 \mathrm{H}, \mathrm{H} 8), 4.07$ (s, 2H, $\left.\underline{\mathrm{C}}_{2} \mathrm{Ph}\right), 7.51$ (ddd, $1 \mathrm{H}, \mathrm{H} 4, J_{4.3} 8.0 \mathrm{~Hz}, J_{4.5} 6.7 \mathrm{~Hz}, J_{4.6} 2.4 \mathrm{~Hz}$ ), 7.68 (m, 2H, H5, H6), 7.97 (d, 1H, H3); ${ }^{13} \mathrm{C} \mathrm{NMR} \mathrm{(50} \mathrm{MHz,} \mathrm{CD} \mathrm{OD):}$ $\delta 40.6\left(\underline{\mathrm{CH}}_{2} \mathrm{NH}_{2}\right) ; 47.5\left(\underline{\mathrm{CH}}_{2} \mathrm{NH}\right) ; 50.4\left(\underline{\mathrm{CH}}_{2} \mathrm{Ph}\right) ; 125.9(\mathrm{C} 3)$; 129.6 (C4); 132.3 (C6); 134.5 (C5); 136.0 (C1); 146.2 (C2).

Data for 8. Oil; Elemental analysis (Found: C, 63.70; H, 7.38; N, 13.41. Calc. for $\mathrm{C}_{11} \mathrm{H}_{16} \mathrm{~N}_{2} \mathrm{O}_{2}: \mathrm{C}, 63.46 ; \mathrm{H}, 7.69 ; \mathrm{N}$, 13.46\%); IR (KBr) $v_{\max } / \mathrm{cm}^{-1}: 3248,3050,2956,2903,1728$, 1286; ' $\mathrm{H}$ NMR (200MHz, DMSO- $\left.d_{6}\right): \delta 2.78(\mathrm{t}, 2 \mathrm{H}, \mathrm{H} 9$, $\left.J_{9-8} 5.6 \mathrm{~Hz}\right), 2.94$ (t, $\left.2 \mathrm{H}, \mathrm{H} 8\right), 3.85$ (s, 3H, OC$\left.{ }_{3}\right), 4.15-$ 4.36 (m, 5H, $\underline{\mathrm{H}}_{2} \mathrm{Ph}, \mathrm{N} \underline{\mathrm{H}}$ e $\left.\underline{\mathrm{H}}_{2}\right), 7.54$ (d, 2H, H2, H6, J 
$8.1 \mathrm{~Hz}), 7.95$ (d, 2H, H3, H5); ${ }^{13} \mathrm{C}$ NMR (50 MHz, DMSO$\left.d_{6}\right): \delta$ 38.2-40.7 (C9), 45.3 (C8), 51.7 (C7), 52.1 (C11), 128.4 (C2), 129.1 (C3), 145.4 (C1), 166.2 (C10).

Data for 16. Oil; Elemental analysis (Found: C, 57.90; $\mathrm{H}, 5.65 ; \mathrm{N}, 16.68$. Calc. for $\mathrm{C}_{16} \mathrm{H}_{18} \mathrm{~N}_{4} \mathrm{O}_{4}: \mathrm{C}, 58.18 ; \mathrm{H}, 5.45$; $\mathrm{N}, 16.97 \%) ; \mathrm{IR}(\mathrm{KBr}) \mathrm{v}_{\max } / \mathrm{cm}^{-1}: 3064,2845,1525,1351$;

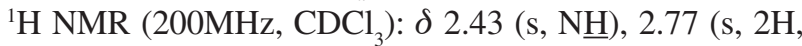
$\mathrm{C}_{2} \mathrm{NH}$ ), 4.03 (s, 2H, $\mathrm{C}_{2} \mathrm{Ph}$ ), 7.41 (ddd, $1 \mathrm{H}, \mathrm{H} 4, J_{4-3} 8.1$ $\left.\mathrm{Hz}, J_{4-5} 6.6 \mathrm{~Hz}, J_{4-6} 2.5 \mathrm{~Hz}\right), 7.55-7.60(\mathrm{~m}, 2 \mathrm{H}, \mathrm{H} 5$ and H6), $7.92(\mathrm{~d}, 1 \mathrm{H}, \mathrm{H} 3) ;{ }^{13} \mathrm{C}$ NMR $\left(50 \mathrm{MHz}, \mathrm{CDCl}_{3}\right): \delta$ $48.7\left(\underline{\mathrm{CH}}_{2} \mathrm{NH}\right), 51.0\left(\underline{\mathrm{C}}_{2} \mathrm{Ph}\right), 124.7(\mathrm{C} 3), 128.1(\mathrm{C} 4)$, 131.2 (C5), 133.1 (C6), 135.5 (C1), 149.1 (C2).

Synthesis of proligands N-(4-aminobenzyl)ethylenediamine (12) and N-(2-aminobenzyl)ethylenediamine (13)

To a solution of compounds $\mathbf{6}$ or $\mathbf{7}(4 \mathrm{mmol})$ in methanol $(10 \mathrm{~mL})$ was added $10 \mathrm{mg}$ of palladium on activated carbon $10 \%$. The reaction mixture was stirred under a hydrogen atmosphere for $10 \mathrm{~h}$ at room temperature. After this time, it was filtered and the solvent was evaporated under reduced pressure, and the residue purified by chromatography column using dichloromethane/methanol as eluent. Yields: 12 (0.52 g; 48\%) and 13 (0.47 g; 43\%).

Data for 12. Oil; Elemental analysis (Found: C, 65.55; $\mathrm{H}, 8.89 ; \mathrm{N}, 25.73$. Calc. for $\mathrm{C}_{9} \mathrm{H}_{15} \mathrm{~N}_{3}: \mathrm{C}, 65.45 ; \mathrm{H}, 9.09$; $\mathrm{N}, 25.45 \%)$; IR (KBr) $v_{\max } / \mathrm{cm}^{-1}: 3410,3344,3217,2922$, 2854; ${ }^{1} \mathrm{H}$ NMR (200 MHz, $\left.\mathrm{D}_{2} \mathrm{O}\right): \delta 3.31-3.37(\mathrm{~m}, 4 \mathrm{H}$, $\left.\mathrm{NHC}_{2} \underline{\mathrm{C}}_{2} \mathrm{NH}_{2}\right), 4.25$ (s, $\left.2 \mathrm{H}, \underline{\mathrm{C}}_{2} \mathrm{Ph}\right), 7.37$ (d, $2 \mathrm{H}, \mathrm{H} 3$, $\left.\mathrm{H} 5, J_{3-2} 8.6 \mathrm{~Hz}\right), 7.52$ (d, 2H, H2, H6); ${ }^{13} \mathrm{C} \mathrm{NMR}(50 \mathrm{MHz}$, $\left.\mathrm{D}_{2} \mathrm{O}\right): \delta 35.4\left(\underline{\mathrm{CH}}_{2} \mathrm{NH}_{2}\right), 43.8\left(\underline{\mathrm{CH}}_{2} \mathrm{NH}\right), 50.7\left(\underline{\mathrm{CH}}_{2} \mathrm{Ph}\right)$, 124.0 (C3/C5), 131.1 (C2/C6), 131.3 (C1), 131.6 (C4).

Data for 13. Oil; Elemental analysis (Found: C, 65.60; H, 9.01; N, 25.76. Calc. for $\mathrm{C}_{9} \mathrm{H}_{15} \mathrm{~N}_{3}: \mathrm{C}, 65.45 ; \mathrm{H}, 9.09 ; \mathrm{N}$, 25.45\%); IR (KBr) $v_{\max } / \mathrm{cm}^{-1}: 3208,2981,1580 ;{ }^{1} \mathrm{H}$ NMR (200 MHz, $\left.\mathrm{D}_{2} \mathrm{O}\right): \delta 3.31\left(\mathrm{t}, 2 \mathrm{H}, \mathrm{H} 9, J_{9-8} 6.3 \mathrm{~Hz}\right), 3.45$ (t, 2H, H8), 4.30 (s, $\left.2 \mathrm{H}, \mathrm{C}_{2} \mathrm{Ph}\right), 7.15-7.52$ (m, 4H, H3, H4, $\mathrm{H} 5, \mathrm{H} 6) ;{ }^{13} \mathrm{C}$ NMR $\left(50 \mathrm{MHz}, \mathrm{D}_{2} \mathrm{O}\right): \delta 35.5\left(\underline{\mathrm{C}}_{2} \mathrm{NH}_{2}\right), 44.2$ $\left(\underline{\mathrm{CH}}_{2} \mathrm{NH}\right), 46.6\left(\underline{\mathrm{CH}}_{2} \mathrm{Ph}\right), 122.6(\mathrm{C} 3), 123.5$ (C5), 128.2 (C6), 131.7 (C4), 131.9 (C1), 132.2 (C2).

Synthesis of proligands 2-[N-(ethylenediamino)-methyl]pyridine (19) and 4-[N-(ethylenediamino)-methyl]pyridine (24)

To a solution of ethylenediamine $(6.7 \mathrm{~mL}, 100 \mathrm{mmol})$ in ethanol $(45 \mathrm{~mL})$, the corresponding (bromomethyl) pyridine hydrobromide $(2.53 \mathrm{~g}, 10 \mathrm{mmol})$ was slowly added during $4 \mathrm{~h}$. The reaction mixture was stirred for 24 $\mathrm{h}$ at room temperature. After this time, the solvent was evaporated under reduced pressure, and the residue purified by chromatography on silica, using dichloromethane/methanol as eluent. Yields: $19(1.10 \mathrm{~g} ; 73 \%)$ and $24(1.20 \mathrm{~g} ; 81 \%)$.

Data for 19. Oil; Elemental analysis (Found: C, 63.38; $\mathrm{H}, 8.53 ; \mathrm{N}, 28.03$. Calc. for $\mathrm{C}_{8} \mathrm{H}_{13} \mathrm{~N}_{3}$ : C, 63.58; $\mathrm{H}, 8.61$; $\mathrm{N}, 27.81 \%) ; \mathrm{IR}(\mathrm{KBr}) \mathrm{v}_{\max } / \mathrm{cm}^{-1}: 3470,3013,2916,2845$, 1596; ${ }^{1} \mathrm{H}$ NMR (200 MHz, $\left.\mathrm{D}_{2} \mathrm{O}\right): \delta 2.77$ (t, 2H, H9, $J_{9-8}$ $6.3 \mathrm{~Hz}$ ), 2.94 (t, 2H, H8), 3.78 (s, $\left.\mathrm{C}_{2} \mathrm{Py}\right), 7.25$ (ddd, 1H, $\left.\mathrm{H} 5, J_{5-6} 5.1 \mathrm{~Hz}, J_{5-4} 7.6 \mathrm{~Hz}, J_{5-3} 1.1 \mathrm{~Hz}\right), 7.36(\mathrm{~d}, 1 \mathrm{H}, \mathrm{H} 3$, $\left.J_{3-4} 7.6 \mathrm{~Hz}\right), 7.74\left(\mathrm{td}, 1 \mathrm{H}, \mathrm{H} 4, J_{4-6} 1.7 \mathrm{~Hz}\right), 8.37(\mathrm{~d}, 1 \mathrm{H}$, H6); ${ }^{13} \mathrm{C}$ NMR $\left(50 \mathrm{MHz}, \mathrm{D}_{2} \mathrm{O}\right): \delta 38.9\left(\mathrm{CH}_{2} \mathrm{NH}_{2}\right), 46.9$ $\left(\underline{\mathrm{CH}}_{2} \mathrm{NH}\right), 53.1\left(\underline{\mathrm{CH}}_{2} \mathrm{Py}\right), 123.3$ (C3), 123.7 (C5), 137.9 (C4), 148.3 (C6), 156.4 (C2).

Data for 24. Oil; Elemental analysis (Found: C, 63.87; $\mathrm{H}, 8.83 ; \mathrm{N}, 27.53$. Calc. for $\mathrm{C}_{8} \mathrm{H}_{13} \mathrm{~N}_{3}: \mathrm{C}, 63.58 ; \mathrm{H}, 8.61$; $\mathrm{N}, 27.81 \%) ; \mathrm{IR}(\mathrm{KBr}) \mathrm{v}_{\max } / \mathrm{cm}^{-1}: 3368,3045,2921,2848$; ${ }^{1} \mathrm{H}$ NMR (200 MHz, $\left.\mathrm{D}_{2} \mathrm{O}\right): \delta 2.56$ (t, 2H, H9, $J_{9-8} 6.0 \mathrm{~Hz}$ ), 2.66 (t, 2H, H8), 3.61 (s, $\left.\underline{\mathrm{C}}_{2} \mathrm{Py}\right), 7.26$ (d, 2H, H3, H5, $\left.J_{3-2} 6.1 \mathrm{~Hz}\right), 8.29(\mathrm{~d}, 2 \mathrm{H}, \mathrm{H} 2, \mathrm{H} 6) ;{ }^{13} \mathrm{C}$ NMR $(50 \mathrm{MHz}$, $\left.\mathrm{D}_{2} \mathrm{O}\right): \delta 40.1\left(\underline{\mathrm{C}}_{2} \mathrm{NH}_{2}\right), 50.1\left(\underline{\mathrm{C}}_{2} \mathrm{NH}\right), 51.5\left(\underline{\mathrm{CH}}_{2} \mathrm{Py}\right)$, 124.1 (C3 and C5); 149.1 (C2 and C6), 149.9 (C4); EIMS (70 eV) m/z (\%): $152\left[\mathrm{M}^{+}+1\right]$ (35); 121 (100); 92 (64).

Synthesis of proligands 2-[N-(2-aminoethanethiol) methyl]-pyridine (20) and 4-[N-(2-aminoethanethiol) methyl]-pyridine (25)

To a solution of the 2-aminoethanethiol hydrochloride $(2.27 \mathrm{~g} ; 20 \mathrm{mmol})$ in ethanol $(50 \mathrm{~mL})$, were added first sodium hydrogencarbonate $(4.20 \mathrm{~g} ; 50 \mathrm{mmol})$ and then slowly, the corresponding (bromomethyl)pyridine hydrobromide $(2.53 \mathrm{~g}, 10 \mathrm{mmol})$ during $4 \mathrm{~h}$. The reaction mixture was stirred for $24 \mathrm{~h}$ at room temperature. After this time, it was filtered and the solvent was evaporated. The residue was purified by column chromatography using dichloromethane/methanol as eluent yielding 20 (1.39 g; $83 \%)$ and 25 (1.31 g; 78\%).

Data for 20. Oil; Elemental analysis (Found: C, 56.87; $\mathrm{H}, 6.88 ; \mathrm{N}, 16.95$. Calc. for $\mathrm{C}_{8} \mathrm{H}_{12} \mathrm{~N}_{2} \mathrm{~S}: \mathrm{C}, 57.14 ; \mathrm{H}, 7.14$; $\mathrm{N}, 16.67 \%)$; IR (KBr) $v_{\max } / \mathrm{cm}^{-1}: 3463,3013,2923,1597$, 632; ${ }^{1} \mathrm{H}$ NMR (200 MHz, $\mathrm{D}_{2} \mathrm{O}$ ): $\delta 2.64$ (t, 2H, H9, $J_{9-8} 6.6$ $\mathrm{Hz}$ ), 3.00 (t, 2H, H8), 3.75 (s, 2H C. $\underline{H}_{2} \mathrm{Py}$ ), 7.21 (ddd, $1 \mathrm{H}$, $\left.\mathrm{H} 5, J_{5-6} 5.1 \mathrm{~Hz}, J_{5-4} 7.7 \mathrm{~Hz}, J_{5-3} 1.1 \mathrm{~Hz}\right), 7.33(\mathrm{~d}, 1 \mathrm{H}, \mathrm{H} 3$, $\left.J_{3-4} 7.7 \mathrm{~Hz}\right), 7.70\left(\mathrm{td}, 1 \mathrm{H}, \mathrm{H} 4, J_{4-5}=J_{5-4}, J_{4-6} 1.7 \mathrm{~Hz}\right) ; 8.32$ (d, 1H, H6); ${ }^{13} \mathrm{C}$ NMR (50 MHz, $\left.\mathrm{D}_{2} \mathrm{O}\right): \delta 28.1\left(\underline{\mathrm{CH}}_{2} \mathrm{SH}\right)$, 
$35.9\left(\underline{\mathrm{CH}}_{2} \mathrm{NH}\right), 38.2\left(\mathrm{CH}_{2} \mathrm{Py}\right), 123.0$ (C3), 124.0 (C5), 138.4 (C4), 148.6 (C6), 159.9 (C2).

Data for 25. Oil; Elemental analysis (Found: C, 56.86; $\mathrm{H}, 6.90 ; \mathrm{N}, 16.82$. Calc. for $\mathrm{C}_{8} \mathrm{H}_{12} \mathrm{~N}_{2} \mathrm{~S}: \mathrm{C}, 57.14 ; \mathrm{H}, 7.14$; $\mathrm{N}, 16.67 \%)$; IR (KBr) $v_{\max } / \mathrm{cm}^{-1}: 3418,3051,2929,2471$, 648; ${ }^{1} \mathrm{H}$ NMR (200 MHz, $\left.\mathrm{D}_{2} \mathrm{O}\right): \delta 2.57$ (t, 2H, H9, $J_{9-8} 6.6$ $\mathrm{Hz}), 2.96$ (t, 2H, H8), 3.64 (s, 2H, $\left.\underline{\mathrm{H}}_{2} \mathrm{Py}\right), 7.25$ (d, $2 \mathrm{H}$, $\left.\mathrm{H} 3, \mathrm{H} 5, J_{3-2} 6.2 \mathrm{~Hz}\right), 8.27$ (d, 2H, H2, H6); ${ }^{13} \mathrm{C}$ NMR (50 $\left.\mathrm{MHz}, \mathrm{D}_{2} \mathrm{O}\right): \delta 28.4\left(\underline{\mathrm{CH}}_{2} \mathrm{SH}\right), 33.5\left(\underline{\mathrm{CH}}_{2} \mathrm{NH}\right), 38.2$ ( $\left.\underline{\mathrm{CH}}_{2} \mathrm{Py}\right), 124.4$ (C3/C5), 148.7 (C4), 148.8 (C2/C6).

\section{Synthesis of the complexes}

To a solution of $\mathrm{K}_{2}\left[\mathrm{PtCl}_{4}\right](0.415 \mathrm{~g}, 1 \mathrm{mmol})$ in water $(4 \mathrm{~mL})$, the appropriate proligand $(1 \mathrm{mmol})$ dissolved in water or methanol $(5 \mathrm{~mL})$ was slowly added with stirring. After $24 \mathrm{~h}$ in the dark at room temperature, the solid formed was filtered off, washed with water and dried. Yields: 2 (0.26 g; 60\%); 9 (0.29 g; 63\%); 10 (0.34 g; 74\%); 11 (0.19 g; 41\%); 14 (0.34 g; 79\%); 15 (0.29 g; 68\%); 17 (0.34 g; 58\%); 21 (0.29 g; 71\%); 22 (0.32 g; 74\%); 26 (0.28 g; 69\%); 27 (0.34 g; 78\%).

Data for 2. Brown solid, mp 203-218 ${ }^{\circ} \mathrm{C}$ (decomp.); Elemental analysis (Found: C, 19.20; H, 1.90; N, 6.30. Calc. for $\mathrm{C}_{7} \mathrm{H}_{7} \mathrm{~N}_{2} \mathrm{Cl}_{2} \mathrm{~F}_{3} \mathrm{Pt}$ : C, 19.00; $\mathrm{H}, 1.58 ; \mathrm{N}, 6.33 \%$ ); IR (KBr) $v_{\max } / \mathrm{cm}^{-1}: 3132,3049,1332,557,325 ;{ }^{1} \mathrm{H}$ NMR (400 MHz, DMSO- $d_{6}$ ): $\delta 7.60$ (m, 3H, H3, H5, H6), 7.82, 7.97, $8.51\left(3 \mathrm{~s}, 4 \mathrm{H}, \mathrm{NH}_{2}\right) ;{ }^{195} \mathrm{Pt}$ NMR (86MHz; DMSO$\left.d_{6}\right): \delta-2194$.

Data for 9. Yellow solid, $\mathrm{mp}>300{ }^{\circ} \mathrm{C}$ (decomp.); Elemental analysis (Found: C, 23.00; H, 2.61; N, 9.00. Calc. for $\mathrm{C}_{9} \mathrm{H}_{13} \mathrm{~N}_{3} \mathrm{Cl}_{2} \mathrm{O}_{2} \mathrm{Pt}$ : C, 23.42; $\left.\mathrm{H}, 2.81 ; \mathrm{N}, 9.11 \%\right)$; ${ }^{1} \mathrm{H}$ NMR (400 MHz, DMSO- $\left.d_{6}\right): \delta 2.11$ (s, 1H, H9a), 2.28 (s, 3H, H8a, H8b and H9b), 4.12 (dd, 1H, H7a, $J_{7 \mathrm{a}-\mathrm{b}} 13.5$ $\left.\mathrm{Hz}, J_{7 \mathrm{a}-\mathrm{NH}} 8.5 \mathrm{~Hz}\right), 4.31$ (d, 1H, H7b), 5.28-5.38 (2s, 2H,

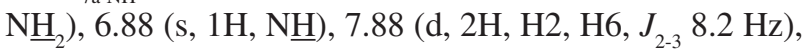
8.24 (d, 2H, H3, H5); ${ }^{13} \mathrm{C}$ NMR (100MHz, DMSO- $d_{6}$ ): $\delta$ $46.5\left(\underline{\mathrm{CH}}_{2} \mathrm{NH}_{2}\right), 54.2\left(\underline{\mathrm{CH}}_{2} \mathrm{NH}\right), 54.6\left(\underline{\mathrm{CH}}_{2} \mathrm{Ph}\right), 123.6(\mathrm{C} 3 /$ C5), 131.4 (C2/C6), 142.8 (C1), 147.2 (C4).

Data for 10. Yellow solid, mp 263-274 ${ }^{\circ} \mathrm{C}$ (decomp.); Elemental analysis (Found: C, 22.95; H, 2.70; N, 9.10. Calc. for $\mathrm{C}_{9} \mathrm{H}_{13} \mathrm{~N}_{3} \mathrm{Cl}_{2} \mathrm{O}_{2} \mathrm{Pt}$ : C, 23.42; H, 2.81; N, 9.11\%); IR (KBr) $v_{\text {max }} / \mathrm{cm}^{-1}: 3292,3218,3072,2970,1524,1357$, 495, 318; ${ }^{1} \mathrm{H}$ NMR (200 MHz, DMSO- $d_{6}$ ): $\delta 2.55$ (m, 4H, $\left.\mathrm{NHC}_{2} \mathrm{C}_{2} \mathrm{NH}_{2}\right), 4.30$ (dd, $1 \mathrm{H}, \mathrm{H} 7 \mathrm{a}, J_{7 \mathrm{a}-7 \mathrm{~b}} 13.8 \mathrm{~Hz}, J_{7 \mathrm{a}-\mathrm{NH}}$ $10.5 \mathrm{~Hz}), 4.94$ (d, 1H, H7b), 6.54 (s, 2H, $\mathrm{N}_{2}$ ), 7.09 (s, $1 \mathrm{H}, \mathrm{N} \underline{\mathrm{H}}), 7.78$ (t, $1 \mathrm{H}, \mathrm{H} 4, J_{4-3} 7.5 \mathrm{~Hz}, J_{4-5} 7.5 \mathrm{~Hz}$ ), 7.81 (t,
1H, H5, $\left.J_{5-6} 7.5 \mathrm{~Hz}\right), 7.99$ (d, 1H, H6), 8.17 (d, 1H, H3); ${ }^{13} \mathrm{C}$ NMR (50 MHz, DMSO- $\left.d_{6}\right): \delta 44.1\left(\underline{\mathrm{CH}}_{2} \mathrm{NH}_{2}\right), 51.2$ $\left(\underline{\mathrm{CH}}_{2} \mathrm{NH}\right), 54.0\left(\underline{\mathrm{CH}}_{2} \mathrm{Ph}\right), 124.9$ (C3), 129.6 (C6), 129.7 (C4), 132.6 (C5), 133.9 (C1), 148.6 (C2).

Data for 11. Yellow solid, mp 261-270 ${ }^{\circ} \mathrm{C}$ (decomp.); Elemental analysis (Found: C, 28.00; H, 3.60; N, 6.10. Calc. for $\mathrm{C}_{11} \mathrm{H}_{16} \mathrm{~N}_{2} \mathrm{Cl}_{2} \mathrm{O}_{2} \mathrm{Pt}$ : C, 27.84; H, 3.37; N, 5.90\%); IR (KBr) $v_{\text {max }} / \mathrm{cm}^{-1}: 3216,3149,3119,2953,2846,1719$, 492, 325; ${ }^{1} \mathrm{H}$ NMR (200MHz, DMSO- $d_{6}$ ): $\delta$ 2.43-2.49 (m, 4H, H8, H9), 3.78 (s, 3H, OC$\underline{H}_{3}$ ), 4.09 (dd, 1H, H7a, $\left.J_{7 \mathrm{a}-7 \mathrm{~b}} 12.1 \mathrm{~Hz}, J_{7 \mathrm{a}-\mathrm{NH}} 8.3 \mathrm{~Hz}\right), 4.28(\mathrm{~d}, 1 \mathrm{H}, \mathrm{H} 7 \mathrm{~b}), 6.08(2 \mathrm{~s}$, $\left.2 \mathrm{H}, \mathrm{N}_{2}\right), 7.43$ (s, 1H, N $\left.\underline{\mathrm{H}}\right), 7.69$ (d, 2H, H2, H6, J $\mathrm{J}_{2-3} 8.2$ $\mathrm{Hz}$ ), 7.93 (d, 2H, H3, H5); ${ }^{13} \mathrm{C}$ NMR (50 MHz, DMSO$\left.d_{6}\right): \delta 30.6\left(\underline{\mathrm{CH}}_{2} \mathrm{NH}_{2}\right), 45.1\left(\underline{\mathrm{CH}}_{2} \mathrm{NH}\right), 52.2\left(\mathrm{OCH}_{3}\right), 54.4$ $\left(\underline{\mathrm{CH}}_{2} \mathrm{Ph}\right), 123.7$ (C4), 129.6 (C2/C6), 130.0 (C3/C5), 139.6 (C1), 165.8 (C10).

Data for 14. Yellow solid, mp 284-288 ${ }^{\circ} \mathrm{C}$ (decomp.); Elemental analysis (Found: C, 24.90; H, 3.08; N, 9.35. Calc. for $\mathrm{C}_{9} \mathrm{H}_{15} \mathrm{~N}_{3} \mathrm{Cl}_{2} \mathrm{Pt}$ : C, 25.05; H, 3.48; N, 9.74\%); IR $(\mathrm{KBr}) v_{\max } / \mathrm{cm}^{-1}: 3204,3111,2952,2892,1610,575,327$, 321; ${ }^{1} \mathrm{H}$ NMR (200MHz, DMSO- $d_{6}$ ): $\delta 2.50(\mathrm{~s}, 4 \mathrm{H}$, $\left.\mathrm{NHC}_{2} \underline{\mathrm{C}}_{2} \mathrm{NH}_{2}\right), 4.02\left(\mathrm{~d}, 1 \mathrm{H}, \mathrm{H} 7 \mathrm{a}, J_{7 \mathrm{a}-\mathrm{7b}} 12.5 \mathrm{~Hz}\right), 4.23$ (d, $1 \mathrm{H}, \mathrm{H} 7 \mathrm{~b}), 5.27\left(\mathrm{~s}, 2 \mathrm{H}, \mathrm{PhNH}_{2}\right), 6.29$ (s, 2H, $\left.\mathrm{NHCH}_{2} \mathrm{CH}_{2} \mathrm{NH}_{2}\right), 6.55\left(\mathrm{~d}, 2 \mathrm{H}, \mathrm{H} 3, \mathrm{H} 5, J_{3-2} 8.0 \mathrm{~Hz}\right), 6.98$ (s, 1H, N $\underline{H}), 7.13$ (d, 2H, H2, H6); ${ }^{13} \mathrm{C}$ NMR (50 MHz, DMSO- $\left.d_{6}\right): \delta 45.2\left(\underline{\mathrm{C}}_{2} \mathrm{NH}_{2}\right), 52.6\left(\underline{\mathrm{CH}}_{2} \mathrm{NH}\right), 54.3$ $\left(\mathrm{CH}_{2} \mathrm{Ph}\right), 113.9$ (C3/C5), 120.9 (C1), 130.6 (C2/C6), 148.8 (C4).

Data for 15. Brown solid, mp 258-260 ${ }^{\circ} \mathrm{C}$ (decomp.); Elemental analysis (Found: C, 24.80; H, 3.10; N, 9.40. Calc. for $\mathrm{C}_{9} \mathrm{H}_{15} \mathrm{~N}_{3} \mathrm{Cl}_{2} \mathrm{Pt}$ : C, 25.05; H, 3.48; N, 9.74\%); IR $(\mathrm{KBr}) \mathrm{v}_{\max } / \mathrm{cm}^{-1}: 3413,3225,3130,2940,2884,1617,483$, 324, 315; ${ }^{1} \mathrm{H}$ NMR (200 MHz, DMSO- $d_{6}$ ): $\delta 2.71$ (s, 4H, $\left.\mathrm{NHC}_{2} \underline{\mathrm{C}}_{2} \mathrm{NH}_{2}\right), 4.07$ (d, 1H, H7b, $\left.J_{7 \mathrm{~b}-7 \mathrm{a}} 12.0 \mathrm{~Hz}\right), 4.39$ (t, $\left.1 \mathrm{H}, \mathrm{H} 7 \mathrm{a}, J_{7 \mathrm{a}-\mathrm{b}} 12.0 \mathrm{~Hz}, J_{7 \mathrm{a}-\mathrm{NH}} 12.0 \mathrm{~Hz}\right), 5.63$ (s, 2H, $\left.\mathrm{PhNH}_{2}\right), 6.74$ (s, 2H, $\left.\mathrm{NHCH}_{2} \mathrm{CH}_{2} \underline{\mathrm{N}}_{2}\right), 7.23$ (d, 1H, H3, $\left.J_{3-4} 6.7 \mathrm{~Hz}\right), 7.36$ (m, 2H, H4, H5), 7.54 (d, 1H, H6, J $7.6 \mathrm{~Hz}), 7.72$ (s, $\left.1 \mathrm{H}, \mathrm{NHCH}_{2} \mathrm{CH}_{2} \mathrm{NH}_{2}\right) ;{ }^{13} \mathrm{C} \mathrm{NMR}(50 \mathrm{MHz}$, DMSO- $\left.d_{6}\right): \delta 46.5\left(\underline{\mathrm{CH}}_{2} \mathrm{NH}_{2}\right), 53.5\left(\underline{\mathrm{CH}}_{2} \mathrm{NH}\right), 56.8$ $\left(\underline{\mathrm{CH}}_{2} \mathrm{Ph}\right), 122.9$ (C3), 126.4 (C5), 130.1 (C6), 130.9 (C4), 131.5 (C1), 138.9 (C2).

Data for 17. Brown solid, mp 243-245 ${ }^{\circ} \mathrm{C}$ (decomp.); Elemental analysis (Found: C, 32.50; H, 3.08; N, 9.65. Calc. for $\mathrm{C}_{16} \mathrm{H}_{18} \mathrm{~N}_{4} \mathrm{Cl}_{2} \mathrm{O}_{4} \mathrm{Pt}$ : C, 32.21; H, 3.02; N, 9.39\%); IR (KBr) $v_{\max } / \mathrm{cm}^{-1}$ : 3262; 3124; 2954; 1524; 1342; 515; 471; 316; ${ }^{1} \mathrm{H}$ NMR (200 MHz, DMSO- $d_{6}$ ): $\delta$ 2.26-2.82 (m, $\left.2 \mathrm{H}, \mathrm{NHC}_{2} \underline{\mathrm{C}}_{2} \mathrm{NH}\right), 4.11(\mathrm{~m}, 1 \mathrm{H}, \mathrm{H} 7 \mathrm{a}), 4.93(\mathrm{~d}, 1 \mathrm{H}$, 
$\left.\mathrm{H} 7 \mathrm{~b}, J_{7 \mathrm{~b}-7 \mathrm{a}} 7.5 \mathrm{~Hz}\right), 6.61(\mathrm{~s}, 1 \mathrm{H}, \mathrm{N} \underline{\mathrm{H}}), 7.65\left(\mathrm{t}, 1 \mathrm{H}, \mathrm{H} 4, J_{4-5}\right.$ $\left.7.5 \mathrm{~Hz}, J_{4-3} 7.5 \mathrm{~Hz}\right), 7.76\left(\mathrm{t}, 1 \mathrm{H}, \mathrm{H} 5, J_{5-6} 7.5 \mathrm{~Hz}\right), 7.78$ (d, 1H, H6), 8.09 (d, 1H, H3); ${ }^{13} \mathrm{C}$ NMR (50 MHz, DMSO$\left.d_{6}\right): \delta 51.8\left(\underline{\mathrm{C}}_{2} \mathrm{NH}\right), 52.8\left(\underline{\mathrm{CH}}_{2} \mathrm{Ph}\right), 124.9(\mathrm{C} 3), 129.4$ (C6), 129.6 (C4), 130.5 (C1), 132.4 (C5), 148.8 (C2).

Data for 21. Yellow solid, mp 274-295 ${ }^{\circ} \mathrm{C}$ (decomp.); Elemental analysis (Found: C, 23.50; H, 3.45; N, 10.51 . Calc. for $\mathrm{C}_{8} \mathrm{H}_{13} \mathrm{~N}_{3} \mathrm{Cl}_{2} \mathrm{Pt}$ : C, 23.02; H, 3.11; N, 10.07\%); IR (KBr) $v_{\text {max }} / \mathrm{cm}^{-1}: 3224,3100,2954,479,314 ;{ }^{1} \mathrm{H}$ NMR $\left(200 \mathrm{MHz}, \mathrm{DMSO}-d_{6}\right): \delta 2.90(\mathrm{~m}, 4 \mathrm{H}, \mathrm{H} 8 \mathrm{a}, \mathrm{H} 8 \mathrm{~b}, \mathrm{H} 9 \mathrm{a}$ H9b), 4.31 (dd, 1H, H7a, $J_{7 \mathrm{a}-7 \mathrm{~b}} 15.4 \mathrm{~Hz}, J_{7 \mathrm{a}-\mathrm{NH}} 4.8 \mathrm{~Hz}$ ), $4.55\left(\mathrm{dd}, 1 \mathrm{H}, \mathrm{H} 7 \mathrm{~b}, J_{7 \mathrm{~b}-\mathrm{NH}} 9.2 \mathrm{~Hz}\right), 5.94$ and $6.17(2 \mathrm{~s}, 2 \mathrm{H}$, $\left.\mathrm{NH}_{2}\right), 7.57(\mathrm{~m}, 1 \mathrm{H}, \mathrm{H} 5), 7.71\left(\mathrm{~d}, 1 \mathrm{H}, \mathrm{H} 3, J_{3-4} 7.7 \mathrm{~Hz}\right)$, 8.09 (s, $1 \mathrm{H}, \mathrm{NH}), 8.19$ (t, $1 \mathrm{H}, \mathrm{H} 4, J_{4-3} 7.7 \mathrm{~Hz}, J_{4-5} 7.7 \mathrm{~Hz}$ ), 8.69 (d, $\left.1 \mathrm{H}, \mathrm{H} 6, J_{6-5} 6.5 \mathrm{~Hz}\right) ;{ }^{13} \mathrm{C}$ NMR $(50 \mathrm{MHz}$, DMSO$\left.d_{6}\right): \delta 51.2\left(\underline{\mathrm{CH}}_{2} \mathrm{NH}_{2}\right), 55.2\left(\underline{\mathrm{CH}}_{2} \mathrm{NH}\right), 57.5\left(\underline{\mathrm{CH}}_{2} \mathrm{Py}\right), 122.9$ (C3), 124.7 (C5), 139.9 (C4), 147.6 (C6), 166.6 (C2).

Data for 22. Yellow solid, $\mathrm{mp}>300{ }^{\circ} \mathrm{C}$ (decomp.); Elemental analysis (Found: C, 21.90; H, 2.20; N, 6.00. Calc. for $\mathrm{C}_{8} \mathrm{H}_{12} \mathrm{~N}_{2} \mathrm{Cl}_{2} \mathrm{PtS}$ : C, 22.11; H, 2.76; N, 6.45\%); IR (KBr) $v_{\max } / \mathrm{cm}^{-1}: 3448,3071,2968,1608,1482,453$, 321,$315 ;{ }^{1} \mathrm{H}$ NMR $\left(200 \mathrm{MHz}\right.$, DMSO- $\left.d_{6}\right): \delta 3.01(\mathrm{~m}, 4 \mathrm{H}$, H8a, H8b, H9a, H9b), 4.75 (d, 1H, H7a, $J_{7 \mathrm{a}-7 \mathrm{~b}} 17.0 \mathrm{~Hz}$ ), 4.82 (d, 1H, H7b), 7.58 (t, 1H, H5, J J $7.2 \mathrm{~Hz}), 7.84$ (d, $\left.1 \mathrm{H}, \mathrm{H} 3, J_{4-3} 7.8 \mathrm{~Hz}\right), 8.15-8.23(\mathrm{~m}, 2 \mathrm{H}, \mathrm{H} 4, \mathrm{NH}), 9.38(\mathrm{~d}$, $1 \mathrm{H}, \mathrm{H} 6) ;{ }^{13} \mathrm{C}$ NMR (50 MHz, DMSO- $\left.d_{6}\right): \delta 34.5\left(\underline{\mathrm{CH}}_{2} \mathrm{SH}\right)$, $38.2\left(\underline{\mathrm{CH}}_{2} \mathrm{NH}\right), 43.6\left(\mathrm{C}_{2} \mathrm{Py}\right), 124.6$ (C3), 125.0 (C5), 140.0 (C4), 149.8 (C2), 162.9 (C6).

Data for 26. Yellow solid, $\mathrm{mp}>300{ }^{\circ} \mathrm{C}$ (decomp.); Elemental analysis (Found: C, 23.00; H, 2.95; N, 9.80. Calc. for $\mathrm{C}_{8} \mathrm{H}_{13} \mathrm{~N}_{3} \mathrm{Cl}_{2} \mathrm{Pt}$ : C, 23.02; H, 3.11; N, 10.07\%); IR (KBr) $v_{\max } / \mathrm{cm}^{-1}$ : 3191, 3104, 2886, 1621, 502, 324.

Data for 27. Yellow solid, $\mathrm{mp}>300{ }^{\circ} \mathrm{C}$ (decomp.); Elemental analysis (Found: C, 22.00; H, 2.51; N, 6.30. Calc. for $\mathrm{C}_{8} \mathrm{H}_{12} \mathrm{~N}_{2} \mathrm{Cl}_{2} \mathrm{PtS}: \mathrm{C}, 22.11 ; \mathrm{H}, 2.76 ; \mathrm{N}, 6.45 \%$ ); IR (KBr) $v_{\max } / \mathrm{cm}^{-1}: 3205,3015,2921,1618,502$.

\section{In vitro cytotoxic screening}

The cytotoxicity studies were done at the laboratory of medical oncology of the Academic Hospital of Rotterdam (The Netherlands). Compound $\mathbf{2}$ was screened in vitro in seven human cancer cell lines, MCF7 and EVSAT (mammary cancers), WiDr (colon cancer), IGROV (ovarian cancer), M19 (melanoma), MEL A498 (renal cancer) and H226 (lung cancer) from aqueous solutions containing $1 \%$ of DMSO, according to a protocol described previously. ${ }^{23,24} \mathrm{MCF} 7$ is estrogen receptor (ER)q/ progesterone receptor (PgR)q and EVSA-T is ERy/PgRy. The cell lines WIDR, M19 MEL, A498, IGROV and H226 belong to the anti-cancer screening panel of the National Cancer Institute, Bethesda, Maryland, USA.

Test and reference compounds were dissolved to a concentration of $250,000 \mathrm{ng} \mathrm{mL}^{-1}$ in full medium, by 20 fold dilution of a stock solution containing $1 \mathrm{mg}$ of compound per $200 \mu \mathrm{L}$ aqueous solutions containing $1 \%$ of dimethylsulfoxide (DMSO) or ethanol using a literature procedure, ${ }^{23,24}$ by the microculture sulforhodamine $\mathrm{B}$ (SRB) test. The experiment was started on day 0 . On day $0,150 \mu \mathrm{L}$ of trypsinized tumour cells $(1,500-2,000$ cells/ well) were plated in 96-wells flat-bottommicrotitre plates (Falcon 3072, BD). The plates were preincubated for 48 $\mathrm{h}$ at $37{ }^{\circ} \mathrm{C}, 8.5 \% \mathrm{CO}_{2}$, to allow the cells to adhere. On day 2 , a threefold dilution sequence of ten steps was made in full medium, starting from the stock solution. Every dilution was used in quadruplicate by adding $50 \mu \mathrm{L}$ to a column of four wells. On day 7 , the incubation was terminated by washing the plate twice with phosphatebuffered saline (PBS). Subsequently, the cells were fixed with $10 \%$ trichloroacetic acid in PBS and placed at $4{ }^{\circ} \mathrm{C}$ for $1 \mathrm{~h}$. After five washings with tap water, the cells were stained for at least 15 min with $0.4 \%$ SRB dissolved in $1 \%$ acetic acid. After staining, the cells were washed with $1 \%$ acetic acid to remove the unbound stain. The plates were air-dried and the bound stain was dissolved in $150 \mu \mathrm{L}$ $10 \mathrm{mmol} \mathrm{L}^{-1}$ Tris-base. The absorbance was read at 540 $\mathrm{nm}$ using an automated microplate reader (Labsystems Multickan MS). Data were used for the construction of concentration-response curves and determination of the ID50 value by using the Deltasoft 3 software.

\section{Conclusions}

This work describes the synthesis and characterization of ten new diamine/ aminethiol proligands and their platinum(II) complexes. These complexes have potential for acting as cytotoxic agents, as was demonstrated by preliminary tests. Further investigation of the biological properties of these compounds is desirable to determine their possible utility as anticancer agents.

\section{Acknowledgments}

J.D.S.C., M.V.A., A.P.S.F. and E.T.C. gratefully acknowledge FAPEMIG, CNPq and CAPES for a fellowship and financial support. M.G. thanks Dr. de Vos, (Pharmachemie) and Mr. H. J. Kolker, Dr. J. Verweij, Prof. Dr. G. Stoter, Dr. J. H. M. Schellens (Laboratory of 
Experimental Chemotherapy and Pharmacology, Department of Medical Oncology, Rotterdam Cancer Institute, NL-3008 AE, Rotterdam, The Netherlands). Dedicated to Dr. Wagner Magno Teles (IN MEMORIAM)

\section{References}

1. Rosenberg, B.; Van Camp, L.; Trosco, J. E.; Mansour, V. H.; Nature 1969, 222, 385.

2. Krakoff, I. H.; Platinum and Other Metal Coordination Compounds in Cancer Chemoterapy: Clinical Aplications of Platinum Complexes; Nicoline, M., Martinus Nijhoff Publishing: Boston, M A, 1988, p. 351.

3. Loehrer, P. J.; Williams, S. D.; Einhorn, L. H.; J. Natl. Cancer Inst. 1988, 80, 1373.

4. Loehrer, P. J.; Einhorn, L. H.; Ann. Inter. Med. 1984, 100, 704.

5. Krakoff, I. H.; Cancer Treat. Resp. 1979, 63, 1623.

6. Vermorken, J. B.; Pinedos, H. M.; Neth. J. Med. 1982, 25, 270.

7. Vonhoff, D. D.; Schilky, R.; Reichert, C. M.; Cancer Treat. Resp. 1979, 63, 1527.

8. Cleare, M. J.; Hoeschele, J. D.; Plat. Met. Rev. 1973, 17, 3.

9. Cleare, M. J.; Hoeschele, J. D.; Bioinorg. Chem. 1973, $2,187$.

10. Bednarski, P.; J. Inorg. Biochem. 1995, 80, 1.

11. Connors, T. A.; Jones, M.; Ross, W. C. J.; Braddock, P. D.; Khokhar, A. R.; Tobe, M. L.; Chem. Biol. Interact. 1972, 5, 415.

12. Gale, G. R.; Loretta, L. M.; Atkins, M.; Walker, E. M.; Smith, A. B.; Meischen, S. J.; Proc. Soc. Exp. Biol. Med. 1973, 142, 1349.

13. Hansch, C.; Venger, B. H.; Panthananickal, A.; J. Med. Chem. 1980, 23, 459.

14. Haghighi, S; McAuliffe, C. A.; Hill, W. E.; Kohl, H. H.; Friedman, M. E.; Inorg. Chim. Acta 1980, 43, 113.
15. Meischem, S. J.; Gale, G. R.; Jake, L. M.; Frangakis, C. J.; Rosenblum, M. G.; Walker, E. M.; Atkins, L. M.; Smith, A. B.; J. Natl. Cancer Inst. 1976, 57, 841.

16. Almeida, A. M. P.; Berlinck, R. G. S.; Hajdu, E.; Quim. Nova 1997, 20, 170.

17. Beaumont, K. P.; McAuliffe, C. A.; Cleare, M. J.; Chem. Biol. Interact. 1976, 14, 179.

18. Paul, A. K.; Srivastava, T. S.; Chavan, S. J.; Chitnis, M. P.; Desai, S.; Rao. K. K.; J. Inorg. Biochem. 1996, 61, 179.

19. Farrel, N. P.; Transition Metal Complexes as Drugs and Chemoterapeutic Agents, Kluwer Academic Publishers: Dordrecht, 1989, p. 18.

20. Almeida, M. V.; Cesar, E. T.; Felício, E. C. A.; Fontes, A. P. S.; Gero, M. R.; J. Braz. Chem. Soc. 2000, 11, 154; Cesar, E. T.; Ph.D. Thesis, Universidade Federal de Minas Gerais, Brazil, 2001.

21. Almeida, M. V.; Cesar, E. T.; Felício, E. C. A.; Fontes, A. P. S.; Berg, R. N.; Filho, J. D. S.; Molecules 2002, 7, 405; Cesar, E. T.; Almeida, M. V.; Fontes, A. P. S.; Maia, E. C. P.; Suillerot, A. G.; Couri, M. R. C.; Felício, E. C. A; J. Inorg. Biochem. 2003, 95, 297.

22. Vollano, J. F.; Al-Baker, S.; Dabrowiak, J. C.; Schurig, J. E.; J. Med. Chem. 1987, 30, 716.

23. De Vita Jr., V. T.; Hellman, S.; Rosenberg, S. A.; Cancer: Principles and Practice of Oncology, $5^{\text {th }}$ ed., Lippincott-Raven Publ.: Philadelphia, 1997.

24. Keepers, Y. P.; Pizao, P. E.; Peters, G. J.; Van Arkotte, J.; Winograd, B.; Pinedo, H. M.; Eur. J. Cancer 1991, 27, 897.

Received: October 6, 2005 Published on the web: August 22, 2006 